\title{
Design of Frequency Response Masking Based Reconfigurable Filter Banks for Software Radio Channelization Using Semidefinite Programming
}

\author{
Chen $\mathrm{Wu}$, Xinzhou Xu, Li Zhao, Cairong Zou \\ Key Laboratory of Underwater Acoustic Signal Processing of Ministry of Education \\ Southeast University \\ Nanjing, China \\ 230129135@seu.edu.cn
}

\begin{abstract}
A new reconfigurable filter bank architecture based on frequency response masking (FRM) was recently proposed for software defined radio (SDR) channelization. However, in previous works all the subfilters were designed separately, which makes the solutions suboptimal. In this paper, we propose an improved design method, which takes all desired reconfiguration modes into consideration. The proposed method takes two steps: firstly an initial solution is obtained by separately designing all the subfilters as in previous works, and then the initial solution is updated by iteratively solving a semidefinite programming (SDP) problem. Simulation results demonstrate that the proposed method offers significantly lower approximation errors compared to the conventional design method.
\end{abstract}

Keywords- Frequency response masking (FRM); software radio channelization; reconfigurable filter banks; semidefinite programming (SDP).

\section{INTRODUCTION}

Low complexity and reconfigurability are the two key requirements of a software defined radio (SDR) channelizer [1]. Recently, a new reconfigurable filter bank architecture based on frequency response masking (FRM) technique was proposed for SDR channelization [2-4]. The FRM technique is well known for its low complexity in designing sharp transition-band finite impulse response (FIR) filters [5]. When incorporating reconfigurability to the FRM filters, low complexity and reconfigurability can be satisfied simultaneously. Another advantage of the FRM based reconfigurable filter banks is that channels of nonuniform bandwidths can be extracted.

Previous works on the FRM based reconfigurable filterbanks mainly focused on its applications and implementations. The designing procedure generally involves two steps: first, obtaining the specifications of all the subfilters, and second, designing all the subfilters with the precalculated specifications using the Parks-McClellan algorithm [6]. However, designing all the subfilters separately is an indirect way, which makes the obtained solutions suboptimal.

For the optimal design of the FRM based reconfigurable filter banks, all the subfilters are expected to be designed directly according to the desired modes. It has been demonstrated that, for the design of reconfigurable coefficient decimation FIR filters, taking all reconfiguration modes into consideration can give better results [7].
However, as the cascade structure of the FRM filter, linear programming (LP) used in [7] will not be appropriate for the optimal design of the FRM based reconfigurable filter banks. In earlier works, semidefinite programming (SDP) [8] has been employed for the optimal design of basic single mode FRM filter [9]. So, in this paper, we propose an improved design method, which takes all desired reconfiguration modes into consideration. The optimal design of the FRM based reconfigurable filter banks can be formulated as an SDP problem. Given a reasonable initial design solution, improved design can be obtained by iteratively solving an SDP problem.

The rest of this paper is organized as follows. Section 2 briefly reviews the technique of the FRM based reconfigurable filter banks as well as the conventional designing method. In Section 3, the proposed design method using SDP is presented. In Section 4, simulation results are illustrated. Finally, conclusions are given in Section 5.

\section{FRM BASED RECONFIGURABLE FILTER BANKS}

The architecture of the FRM based reconfigurable filter banks is shown in Fig. 1. Reconfigurability is achieved by reusing the same coefficients of the modal filter $H_{a}(z)$ with different up-sampling factors $M_{i}$. As all the subfilters involved in the FRM based reconfigurable filter banks have wide transition-band, the overall filter lengths can be short and consequently the implementation complexity can be reduced. Moreover, channels of nonuniform bandwidths can be extracted with different $M_{i}$, which is impossible for the discrete Fourier transform based filter banks [2].

The transfer function of each desired reconfiguration mode is given by [5][9]:

$\mathrm{H}_{i}(\mathrm{z})=\mathrm{H}_{a}\left(\mathrm{z}^{M_{i}}\right) \mathrm{H}_{m a i}(\mathrm{z})+\left[\mathrm{z}^{-0.5 M_{i}(\mathrm{Na}-1)}-\mathrm{H}_{a}\left(\mathrm{z}^{M_{i}}\right)\right] \mathrm{H}_{m c i}(\mathrm{z})$

where

$$
\begin{aligned}
& \mathrm{H}_{a}(\mathrm{z})=\sum_{n=0}^{N_{a}-1} h_{a}(\mathrm{n}) \mathrm{z}^{-n} \\
& \mathrm{H}_{m a i}(\mathrm{z})=\sum_{n=0}^{N_{m a i}-1} h_{m a i}(\mathrm{n}) \mathrm{z}^{-n} \\
& \mathrm{H}_{m c i}(\mathrm{z})=\sum_{n=0}^{N_{m c i}-1} h_{m c i}(\mathrm{n}) \mathrm{z}^{-n}
\end{aligned}
$$




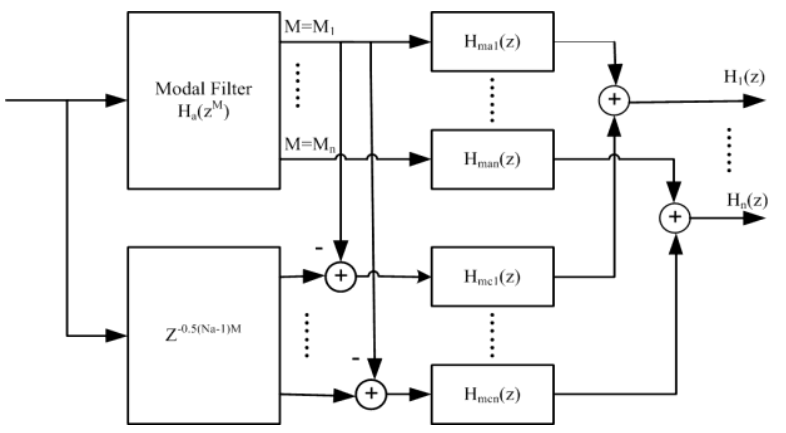

Figure.1 FRM based reconfigurable filter banks.

If the length of each pair of the two masking filters $H_{\text {mai }}(z)$ and $H_{m c i}(z)$ are both even or both odd and $\left(N_{a}-1\right) M_{i}$ is even, each desired mode will have a linear phase response. Without loss of generality, each desired mode can be treated as a zero-phase FIR filter expressed by

$$
\begin{aligned}
& \mathrm{H}_{i}(\omega, \mathbf{x})=\left[\mathbf{a}_{a}^{T} \mathbf{c}_{a i}(\omega)\right]\left[\mathbf{a}_{m a i}^{T} \mathbf{c}_{m a i}(\omega)-\mathbf{a}_{m c i}^{T} \mathbf{c}_{m c i}(\omega)\right]+\mathbf{a}_{m c i}^{T} \mathbf{c}_{m c i}(\omega) \\
& \mathbf{a}_{a}=\left\{\begin{array}{c}
{\left[h_{a}\left(\left(N_{a}-1\right) / 2\right) h_{a}\left(\left(N_{a}+1\right) / 2\right) \cdots h_{a}\left(N_{a}-1\right)\right]^{T}, \text { if } N_{a} \text { odd }} \\
{\left[h_{a}\left(N_{a} / 2\right) \cdots h_{a}\left(N_{a}-1\right)\right]^{T}, \text { if } N_{a} \text { even }}
\end{array}\right. \\
& \mathbf{a}_{m a i}=\left\{\begin{array}{c}
{\left[h_{m a i}\left(\left(N_{m a i}-1\right) / 2\right) h_{m a i}\left(\left(N_{m a i}+1\right) / 2\right) \cdots h_{m a i}\left(N_{m a i}-1\right)\right]^{T}, \text { if } N_{m a i} \text { odd }} \\
{\left[h_{m a i}\left(N_{m a i} / 2\right) \cdots h_{m a i}\left(N_{m a i}-1\right)\right]^{T}, \text { if } N_{m a i} \text { even }}
\end{array}\right. \\
& \mathbf{a}_{m c i}=\left\{\begin{array}{c}
{\left[h_{m c i}\left(\left(N_{m c i}-1\right) / 2\right) h_{m c i}\left(\left(N_{m c i}+1\right) / 2\right) \cdots h_{m c i}\left(N_{m c i}-1\right)\right]^{T}, \text { if } N_{m c i} \text { odd }} \\
{\left[h_{m c i}\left(N_{m c i} / 2\right) \cdots h_{m c i}\left(N_{m c i}-1\right)\right]^{T}, \text { if } N_{m c i} \text { even }}
\end{array}\right. \\
& \mathbf{c}_{a i}(\omega)=\left\{\begin{array}{l}
{\left[12 \cos \left(M_{i} \omega\right) \cdots 2 \cos \left(\left(N_{a}-1\right) M_{i} \omega / 2\right)\right]^{T}, \text { if } N_{a} \text { odd }} \\
2\left[\cos \left(M_{i} \omega / 2\right) \cdots \cos \left(\left(N_{a}-1\right) M_{i} \omega / 2\right)\right]^{T} \text {, if } N_{a} \text { even }
\end{array}\right. \\
& \mathbf{c}_{\text {mai }}(\omega)=\left\{\begin{array}{l}
{\left[12 \cos \left(M_{i} \omega\right) \cdots 2 \cos \left(\left(N_{\text {mai }}-1\right) M_{i} \omega / 2\right)\right]^{T}, \text { if } N_{\text {mai }} \text { odd }} \\
2\left[\cos \left(M_{i} \omega / 2\right) \cdots \cos \left(\left(N_{\text {mai }}-1\right) M_{i} \omega / 2\right)\right]^{T}, \text { if } N_{\text {mai }} \text { even }
\end{array}\right. \\
& \mathbf{c}_{m c i}(\omega)=\left\{\begin{array}{l}
{\left[12 \cos \left(M_{i} \omega\right) \cdots 2 \cos \left(\left(N_{m c i}-1\right) M_{i} \omega / 2\right)\right]^{T}, \text { if } N_{m c i} \text { odd }(3 \mathrm{~g})} \\
2\left[\cos \left(M_{i} \omega / 2\right) \cdots \cos \left(\left(N_{m c i}-1\right) M_{i} \omega / 2\right)\right]^{T}, \text { if } N_{m c i} \text { even }
\end{array}\right.
\end{aligned}
$$

Suppose there are $n$ desired modes with passband edges $\omega_{p 1}, \cdots, \omega_{p n}$ and stopband edges $\omega_{s 1}, \cdots, \omega_{s n}$, respectively. The general procedure to design the FRM based reconfigurable filter banks in previous works is as follows [2-4]:

1. Choose an appropriate modal filter with the passband edge $\omega_{a p}$ and the stopband edge $\omega_{a s}$. The passband ripple and the stopband ripple of the modal filter should satisfy the most stringent requirement of the desired modes.

2. Because the passband edge $\omega_{a p}$ and the stopband edge $\omega_{a s}$ of the modal filter $H_{a}(z)$ remain unchanged for all desired mode. The up-sampling factor $M_{i}$ of the $i$ th mode can be obtained via

$\omega_{a p}=\omega_{p 1} M_{1}-2 \pi m_{1}=\cdots=\omega_{p n} M_{n}-2 \pi m_{n}$

$\omega_{a s}=\omega_{s 1} M_{1}-2 \pi m_{1}=\cdots=\omega_{s n} M_{n}-2 \pi m_{n}$

where $m_{i}=\left\lfloor\omega_{p i} M_{i} /(2 \pi)\right\rfloor$ denotes the largest integer less than $\omega_{p i} M_{i} /(2 \pi)$.

3. The passband edge $\omega_{\text {mapi }}$ and the stopband edge $\omega_{\text {masi }}$ of the masking filter $H_{m a i}$ of the $i$ th mode can be obtained by $\omega_{\text {mapi }}=\omega_{p i}, \omega_{\text {masi }}=\left[2\left(m_{i}+1\right) \pi-\omega_{a s}\right] / M_{i}$

and the passband edge $\omega_{m c p i}$ and the stopband edge $\omega_{m c s i}$ of the complementary masking filter

$H_{m c i}$ of the $i$ th mode can be obtained by

$\omega_{m c p i}=\left(2 m_{i} \pi-\omega_{a p}\right) / M_{i}, \omega_{m c s i}=\omega_{s i}$

4. The length of each of the subfilters can be obtained by

$$
N=-\frac{4 \pi \log _{10}\left(10 \delta_{p} \delta_{s}\right)}{3\left(\omega_{s}-\omega_{p}\right)}-1
$$

where $\delta_{p}$ is the peak passband ripple, $\delta_{s}$ is the peak stopband ripple and $\omega_{s}-\omega_{p}$ is the normalized transition width [10].

5. After the specifications of all the subfilters have been obtained, previous works separately design the subfilters using the conventional ParksMcClellan algorithm [6].

We also notice that there exists another FRM based reconfigurable channel filter for handset applications, which can extract a single channel at a given time [11, 12]. Since the coefficients of all the subfilters of the two architectures are calculated in the same way, the proposed method can also be applied to designing these channelizers.

\section{OUR PROPOSED METHOD}

Although previous designing method can give acceptable results in applications [2-4], designing all the subfilters separately is an indirect way, which leads to a suboptimal solution. We therefore consider improving the design results by directly taking all desired modes into consideration. This basic idea is motivated by [7] that taking all desired modes into consideration can give better results for the design of reconfigurable coefficient decimation FIR filters. However, as the cascade structure of the FRM filters, linear programming (LP) used in [7] will not be appropriate for the optimal design of the FRM based reconfigurable filter banks. Alternatively, we formulate the optimal design of the FRM based reconfigurable filter banks as an SDP problem. Given a reasonable initial design solution, improved design can be obtained by iteratively solving the SDP problem.

The problem of optimal design of the FRM based reconfigurable filter banks taking all desired modes into consideration with the weighted minimax error criterion can be formulated as follows:

minimize $\eta$

subject to $\mathrm{W}_{i}(\omega)^{2}\left|\mathrm{H}_{i}(\omega, \mathbf{x})-\mathrm{H}_{d i}(\omega)\right|^{2} \leq \eta$ for $\omega \in \Omega_{i}, \forall i(8 \mathrm{~b})$ where $\eta$ an upper bound of the error of all modes, $\mathbf{x}=\left[\mathbf{a}_{a}^{T}, \mathbf{a}_{m a l}^{T}, \mathbf{a}_{m c l}^{T}, \cdots, \mathbf{a}_{m a n}^{T}, \mathbf{a}_{m c n}^{T}\right]^{T}$ is a vector that contains the coefficients of the subfiltes defined in (3), $\Omega_{i}=\left[\omega_{1 i}, \cdots, \omega_{N i}\right]$ is a set of dense grid points in the $i$ th desired frequency bands of interest, $\mathrm{H}_{i}(\omega, \mathbf{x})$ is the frequency response of the 
$i$ th desired mode defined in (3), $\mathrm{H}_{d i}(\omega)$ is the desired frequency response of $i$ th mode, which is defined as

$$
\mathrm{H}_{d i}(\omega)=\left\{\begin{array}{lll}
1 & \text { for } & 0 \leq \omega \leq \omega_{p i} \\
0 & \text { for } & \omega_{s i} \leq \omega \leq \pi
\end{array}\right.
$$

and $\mathrm{W}_{i}(\omega)$ is the weighting function defined as

$$
\mathrm{W}_{i}(\omega)=\left\{\begin{array}{ccc}
\delta_{p} / \delta_{s} & \text { for } & 0 \leq \omega \leq \omega_{p i} \\
1 & \text { for } & \omega_{s i} \leq \omega \leq \pi
\end{array}\right.
$$

In order to solve the problem (8), we use an iterative method, which takes an SDP solver in each iteration. Suppose we now have a reasonable solution $\mathbf{x}_{0}$ to start, and we are now in the $k$ th iteration. In a vicinity of $\mathbf{x}_{k}$, we can express $\mathrm{H}_{i}(\omega, \mathbf{x})$ as

$$
\mathrm{H}_{i}\left(\omega, \mathbf{x}_{k}+\boldsymbol{\delta}\right)=\mathrm{H}_{i}\left(\omega, \mathbf{x}_{k}\right)+\mathbf{g}_{i}^{T}\left(\omega, \mathbf{x}_{k}\right) \boldsymbol{\delta}+o(\|\boldsymbol{\delta}\|)
$$

where $\mathbf{g}_{i}\left(\omega, \mathbf{x}_{k}\right)$ is the gradient of $\mathrm{H}_{i}(\omega, \mathbf{x})$ with respect to $\mathbf{X}$ and evaluated at $\mathbf{x}_{k}$, which is defined as:

$$
\begin{aligned}
& \mathbf{g}_{i}\left(\omega, \mathbf{x}_{k}\right)=[\left\{\left[\mathbf{a}_{m a i}^{T} \mathbf{c}_{m a i}(\omega)-\mathbf{a}_{m c i}^{T} \mathbf{c}_{m c i}(\omega)\right] \mathbf{c}_{a}(\omega)\right\}^{T} \overbrace{\mathbf{0}}^{2 i-2} \mathbf{0} \quad \\
& \left\{\left[\mathbf{a}_{a}^{T} \mathbf{c}_{a}(\omega)\right] \mathbf{c}_{m a i}(\omega)\right\}^{T} \quad\left\{\left[1-\mathbf{a}_{a}^{T} \mathbf{c}_{a}(\omega)\right] \mathbf{c}_{m c i}(\omega)\right\}^{T} \quad \overbrace{}^{2 n} \cdots \mathbf{0}
\end{aligned}
$$

Hence, provided the $\|\boldsymbol{\delta}\|$ is small, with $\mathbf{x}=\mathbf{x}_{k}+\boldsymbol{\delta}$ we get

$$
\mathrm{W}_{i}(\omega)\left|\mathrm{H}_{i}(\omega, \mathbf{x})-\mathrm{H}_{d i}(\omega)\right| \approx\left|\mathbf{g}_{i k}^{T}(\omega) \boldsymbol{\delta}+\mathbf{e}_{i k}(\omega)\right|
$$

where

$$
\mathbf{e}_{i k}(\omega)=\mathrm{W}_{i}(\omega)\left[\mathrm{H}_{i}\left(\omega, \mathbf{x}_{k}\right)-\mathrm{H}_{d i}(\omega)\right]
$$

$\mathbf{g}_{i k}(\omega)=\mathrm{W}_{i}(\omega) \mathbf{g}_{i}\left(\omega, \mathbf{x}_{k}\right)$.

From (13), an approximate solution of (8) in the $k$ th iteration can be obtained by solving the following problem:

minimize $\eta$

subject to $\left[\mathbf{g}_{i k}^{T}(\omega) \boldsymbol{\delta}+\mathbf{e}_{i k}(\omega)\right]^{2} \leq \eta$ for $\omega \in \Omega_{i}, \forall i$ (14b)

$$
\|\boldsymbol{\delta}\|^{2} \leq b
$$

where $b$ is a prescribed bound to control the magnitude of $\boldsymbol{\delta}$.

The constraint in (14b) holds if and only if

$$
\hat{\Phi}_{i k}(\eta, \boldsymbol{\delta}, \omega)=\left[\begin{array}{cc}
\eta & \mathbf{g}_{i k}^{T}(\omega) \boldsymbol{\delta}+\mathbf{e}_{i k}(\omega) \\
\mathbf{g}_{i k}^{T}(\omega) \boldsymbol{\delta}+\mathbf{e}_{i k}(\omega) & 1
\end{array}\right] \geq 0
$$

and the constraint in (14c) holds if and only if

$$
\Psi(\boldsymbol{\delta})=\left[\begin{array}{cc}
b & \boldsymbol{\delta}^{T} \\
\boldsymbol{\delta}^{T} & \mathbf{I}_{n}
\end{array}\right] \geq 0
$$

where $\mathbf{I}_{n}$ represents the identity matrix of dimension $n$, and $n$ equals to the dimension of $\mathbf{x}$.

Now we can define an augmented vector $\boldsymbol{\mu}=\left[\begin{array}{ll}\eta & \boldsymbol{\delta}^{T}\end{array}\right]^{T}$ and let $\mathbf{c}=\left[\begin{array}{llll}1 & 0 & \cdots & 0\end{array}\right]^{T}$, the problem in (14) can be formulated as

$$
\begin{array}{ll}
\text { minimize } & \mathbf{c}^{\mathbf{T}} \boldsymbol{\mu} \\
\text { subject to } & \mathbf{G}_{k}(\boldsymbol{\mu}) \geq 0
\end{array}
$$

where

$$
\begin{gathered}
\mathbf{G}_{k}(\boldsymbol{\mu})=\operatorname{diag}\left\{\Phi_{1 k}(\eta, \boldsymbol{\delta}), \cdots, \Phi_{n k}(\eta, \boldsymbol{\delta}), \Psi(\boldsymbol{\delta})\right\} \\
\Phi_{i k}(\eta, \boldsymbol{\delta})=\operatorname{diag}\left\{\hat{\Phi}_{i k}\left(\eta, \boldsymbol{\delta}, \omega_{1 i}\right), \cdots, \hat{\Phi_{i k}}\left(\eta, \boldsymbol{\delta}, \omega_{N i}\right)\right\} .
\end{gathered}
$$

Now matrix $\mathbf{G}_{k}(\boldsymbol{\mu})$ in (17b) is affine with respect to vector $\boldsymbol{\mu}$ and the problem in (17) is an SDP problem.
Once the problem of (17) is solved with a minimizer

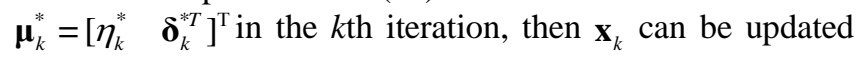
iteratively by $\mathbf{x}_{k+1}=\mathbf{x}_{k}+\boldsymbol{\delta}_{k}^{*}$. The iteration terminates when $\left\|\boldsymbol{\delta}_{k}^{*}\right\|$ becomes insignificant compared to a prescribed tolerance tol.

The general procedure of our proposed design method is listed as follows:

Step 1: Choose an appropriate mode filter with specifications of $\omega_{a p}$ and $\omega_{a s}$.

Step 2: Solve (4) to get the up-sampling factor $M_{i}$ of the $i$ th desired mode, and then obtain the specifications of each pair of the masking filters using (5) and (6).

Step 3: Obtain the length of all the subfilters using (7).

Step 4: Design all the subfilters with the precalculated specifications using the Parks-McClellan algorithm for the initial solution $\mathbf{x}_{0}$. Set $k=0$.

Step 5: Solve (17) with $\mathbf{x}_{k}$ to obtain a minimizer $\boldsymbol{\mu}_{k}^{*}=\left[\begin{array}{ll}\eta_{k}^{*} & \boldsymbol{\delta}_{k}^{* \mathrm{~T}}\end{array}\right]^{\mathrm{T}}, \mathbf{x}_{k+1}=\mathbf{x}_{k}+\boldsymbol{\delta}_{k}^{*}$.

Step 6: if $\left\|\boldsymbol{\delta}_{k}^{*}\right\| \leq t o l$ stop; otherwise $k=k+1$, go to Step 5 .

\section{DESIGN EXAMPLE}

Design example taken in this paper is adopted from [2]. The specifications of the modal filter are $\omega_{a p}=0.4 \pi$, $\omega_{a s}=0.6 \pi$ and $N_{a}=21$. The specifications of all masking filters and the desired filters are shown in Table 1. The weighting factors are selected as unity weights in both the passband and stopband. For each mode, a total of 512 points are uniformly placed in the desired frequency bands of interest. The value of bound $b$ in constraint (14c) should be proportional to the dimension of vector $\mathbf{x}$ [9]. In this paper, we set $b$ as 0.35 and $t o l$ as 0.0005 . We use the Matlab LMI toolbox [13] to solve the SDP problem (17).

TABLE I. SPECIFICATIONS OF SUBFILTERS AND THE DESIRED FILTERS

\begin{tabular}{|l|l|l|l|l|l|l|l|l|}
\hline $\boldsymbol{M}$ & \multicolumn{3}{|l|}{$\boldsymbol{H}(\boldsymbol{z})$} & \multicolumn{2}{l|}{$\boldsymbol{H}_{\text {ma }}(\boldsymbol{z})$} & $\boldsymbol{H}_{m c}(z)$ & & \\
\hline & $\omega_{p}$ & $\omega_{s}$ & $\omega_{\text {map }}$ & $\omega_{\text {mas }}$ & $\mathrm{N}_{\text {ma }}$ & $\omega_{\text {mcp }}$ & $\omega_{\text {mcs }}$ & $\mathrm{N}_{\mathrm{mc}}$ \\
\hline 2 & $0.2 \pi$ & $0.3 \pi$ & $0.2 \pi$ & $0.35 \pi$ & 8 & $\mathrm{NA}$ & $\mathrm{NA}$ & $\mathrm{NA}$ \\
\hline 3 & $0.8 \pi$ & $0.866 \pi$ & $0.8 \pi$ & $\pi$ & 12 & $0.543 \pi$ & $0.866 \pi$ & 12 \\
\hline 4 & $0.6 \pi$ & $0.65 \pi$ & $0.6 \pi$ & $0.85 \pi$ & 16 & $0.4 \pi$ & $0.65 \pi$ & 16 \\
\hline 6 & $0.4 \pi$ & $0.434 \pi$ & $0.4 \pi$ & $0.568 \pi$ & 26 & $0.268 \pi$ & $0.434 \pi$ & 26 \\
\hline
\end{tabular}

The magnitude responses and detailed passband ripples of both the proposed design method as well as the conventional method used in previous works are shown in Fig. 2 and Fig. 3, respectively. As can be clearly seen, the proposed method can produce filters with lower minimax approximation error. The maximum passband ripples and the minimum stopband attenuation of the two design methods for each mode are tabulated in Table 2. It is observed that, our proposed method significantly outperforms the previous design method. Hence, as can be expected, our proposed method can give improved performance for SDR channelization. 
TABLE II. SPECIFICATIONS OF SUBFILTERS AND THE DESIRED FILTERS

\begin{tabular}{|l|l|l|l|l|}
\hline $\boldsymbol{M}$ & \multicolumn{2}{|c|}{$\begin{array}{l}\text { maximum } \\
\text { ripples }(\mathbf{d B})\end{array}$} & \multicolumn{1}{c|}{ passband } & \multicolumn{2}{l|}{$\begin{array}{l}\text { minimum } \\
\text { attenuation(dB) }\end{array}$} \\
\hline & $\begin{array}{l}\text { conventiona } \\
\text { l method }\end{array}$ & $\begin{array}{l}\text { our } \\
\text { proposed }\end{array}$ & $\begin{array}{l}\text { conventional } \\
\text { method }\end{array}$ & $\begin{array}{l}\text { our } \\
\text { proposed }\end{array}$ \\
\hline 2 & 0.1354 & 0.0694 & -40.3001 & -41.9793 \\
\hline 3 & 0.2668 & 0.0694 & -32.7965 & -41.9793 \\
\hline 4 & 0.1508 & 0.0692 & -33.1474 & -42.0102 \\
\hline 6 & 0.1322 & 0.0546 & -36.8437 & -44.2389 \\
\hline
\end{tabular}

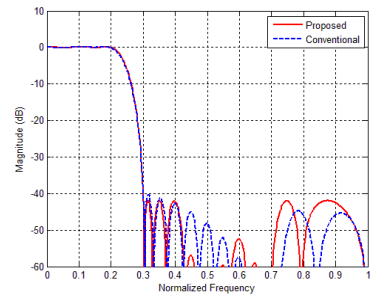

(a)

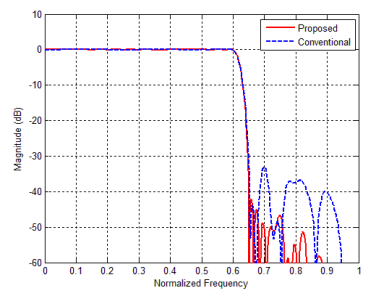

(c)

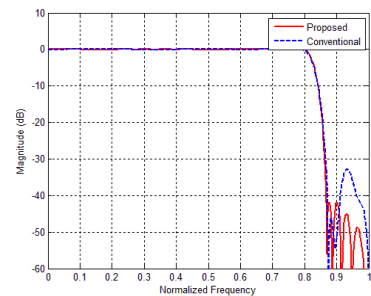

(b)

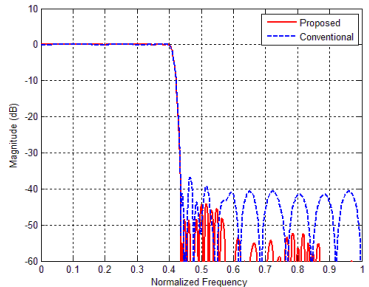

(d)
Figure.2 Magnitude responses of our proposed method and conventional method. (a) $\mathrm{M}=2$; (b) $\mathrm{M}=3$; (c) $\mathrm{M}=4$; (d) $\mathrm{M}=6$.

\section{CONCLUSION}

In this paper, an optimal approach for the design of the FRM based reconfigurable filter banks for SDR channelization is proposed, which takes all desired reconfiguration modes into consideration. The proposed method mainly takes two steps: firstly an initial solution by separately designing the subfilters is obtained as in previous works, and then the initial solutions are improved by iteratively solving an SDP problem. Simulation results demonstrate that the proposed method offers significantly lower minimax approximation errors compared to the conventional design method. As can be expected, our proposed method can give improved performance for SDR channelization.

\section{ACKNOWLEDGMENT}

This work was supported in part by the National Science Foundation of China under Grant No. 61273266, No. 61375028 and No. 61231002.

\section{REFERENCES}

[1] J. Mitola, Software Radio Architecture, Wiley, 2000.

[2] R. Mahesh and A. P. Vinod, "Reconfigurable low area complexity filter bank architecture based on frequency response masking for nonuniform channelization in software radio receivers," IEEE Transactions on Aerospace and Electronic Systems, Vol. 47, pp. 1241 -1255, April 2011.

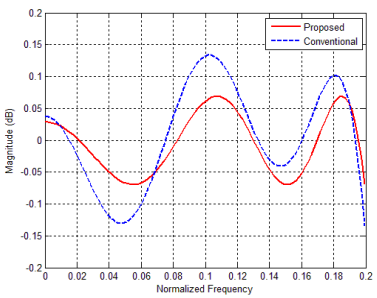

(a)

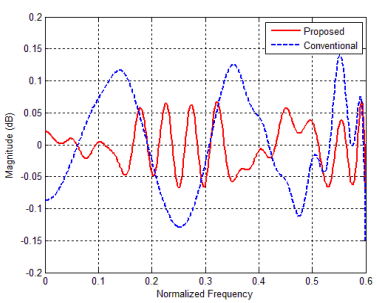

(c)

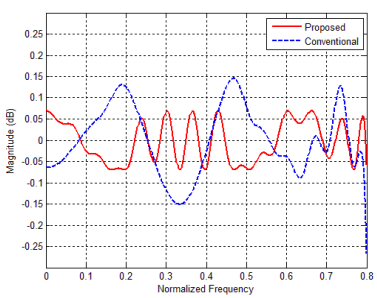

(b)

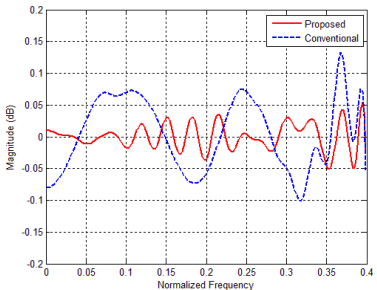

(d)
Figure.3 Passband ripples of our proposed method and conventional method. (a) $\mathrm{M}=2$; (b) $\mathrm{M}=3$; (c) $\mathrm{M}=4$; (d) $\mathrm{M}=6$.

[3] R.Mahesh, A. P. Vinod, E. K. Lai and A. Omondi, "Filter bank channelizers for multi-standard software defined radio receivers," Journal of Signal Processing Systems, vol. 62, pp. 157-171, February 2011.

[4] R. Mahesh and A. P. Vinod, "An area-efficient non-uniform filter bank for low overhead reconfiguration of multi-standard software radio channelizers," Journal of Signal Processing Systems, vol. 64, pp. 413-428, March 2011,

[5] Y. C. Lim, "Frequency-response masking approach for the synthesis of sharp linear phase digital filters," IEEE Transactions on Circuits and Systems, vol. 33, pp. 357-364, April 1986.

[6] T. Parks and J. McClellan, "Chebyshev approximation for nonrecursive digital filters with linear phase," IEEE Transactions on Circuit Theory, vol. 19, pp. 189-194, February 1972.

[7] Z. U. Sheikh and O. Gustafsson, "Linear programming design of coefficient decimation fir filters," IEEE Transactions on Circuits and Systems II: Express Briefs, vol. 59, pp. 60-64, January 2012.

[8] L. Vandenberghe and S. Boyd, "Semidefinite programming," SIAM Review, vol. 38, pp. 49-95, January 1996.

[9] W. S. Lu and T. Hinamoto, "Optimal design of frequency-responsemasking filters using semidefinite programming," IEEE Transactions on Circuits and Systems I: Fundamental Theory and Applications, vol. 50, pp. 557-568, April 2003.

[10] M.Bellanger, "On computational complexity in digital filters," in Peoceedings of the European Conference on Circuit Theory and Design, pp. 58-63, 1981.

[11] R. Mahesh and A. P. Vinod, "Rconfigurable frequency response masking filters for software radio channelization," IEEE Transactions on Circuits and Systems II: Express Briefs, vol. 55, pp. 274-278, March 2008.

[12] K. G. Smitha and A. P. Vinod, "A new low complexity reconfigurable channel filter architecture for software radio handsets," IEEE Singapore International Conference on Communication Systems, pp. 1334-1338, 2008.

[13] P. Gahinet, A. Nemirovskii, A. J. Laub and M. Chilali, "The $1 \mathrm{mi}$ control toolbox," Proceedings of the 33rd IEEE Conference on Decision and Control, pp. 2038-2041, 1994. 